\title{
MiR-99a suppresses cell invasion and metastasis in nasopharyngeal carcinoma through targeting HOXAI
}

This article was published in the following Dove Press journal:

OncoTargets and Therapy

10 February 2017

Number of times this article has been viewed

\author{
Jian-Gang Wang ${ }^{1,2}$ \\ Wu-Ping Tang ${ }^{2}$ \\ Ming-Chu Liao' \\ Yan-Ping Liu' \\ Xiao-Hong $\mathrm{Ai}^{1}$
}

'Department of Radiation Oncology,

The First Affiliated Hospital of

University of South China, Hengyang,

${ }^{2}$ Department of Oncology, Shaoyang

Hospital of TCM, Shaoyang, People's

Republic of China
Correspondence: Xiao-Hong Ai

Department of Radiation Oncology,

The First Affiliated Hospital of University

of South China, 69 Chuanshan Road,

Hengyang 42 I00I, People's Republic

of China

Tel +867348279467

Fax +867348279467

Email I163504926@qq.com
Background: Recent studies reported that miRNAs play important roles in the carcinogenesis and progression of nasopharyngeal carcinoma (NPC). Therefore, further studies are warranted to better elucidate the function and mechanism of miRNAs in NPC.

Methods: Quantitative reverse transcription-polymerase chain reaction (RT-PCR) was used to detect the miR-99a expression in NPC cell lines and tissue samples. Wound healing, transwell migration and invasion, and lung metastatic colonization assays were performed to determine NPC cell migratory, invasive and metastatic abilities of NPC cells. Luciferase reporter assays, quantitative RT-PCR and Western blotting were used to validate the target of miR-99a.

Results: We found that miR-99a was significantly downregulated in NPC cell lines and tissue samples. Ectopic overexpression of miR-99a significantly inhibited NPC cell migration and invasion in vitro, and suppressed lung macroscopic and microscopic metastatic colonization in vivo. Conversely, silencing of miR-99a significantly promoted the migratory and invasive abilities of NPC cells. Furthermore, HOXA1 was validated as a direct target of miR-99a, and ectopic expression of $H O X A 1$ could rescue the suppressive effect of miR-99a overexpression on NPC cell migration and invasion.

Conclusion: Together, these results indicated that miR-99a could inhibit NPC invasion and metastasis by targeting HOXA1, thus providing a novel potential target for miRNA-based treatment for NPC patients in the future.

Keywords: miR-99a, homeobox A1, nasopharyngeal carcinoma, invasion, metastasis

\section{Introduction}

Nasopharyngeal carcinoma (NPC) is a common head and neck malignant tumor with the highest prevalence in South China, especially in Guangdong, Guangxi, Hunan, Fujian, and Jiangxi Provinces, and Hong Kong Special Administrative Region. ${ }^{1}$ Nowadays, with the implement of multidisciplinary combined therapy, such as intensity-modulated radiotherapy, concurrent chemotherapy and target therapy, the 5-year survival of NPC patients has been improved significantly. ${ }^{2}$ However, there were still nearly $30 \%$ of NPC patients eventually developed treatment failure, which was mainly due to distant metastasis. ${ }^{3}$ Therefore, there is a great need to better understand the molecular mechanisms involved in NPC metastasis and to develop personalized therapeutic strategies for patients with NPC.

MicroRNAs (miRNAs) are small noncoding RNAs $~ 19-25$ nucleotides, and they are highly conserved in eukaryotic. ${ }^{4}$ It has been reported that the main function of miRNAs is to negatively regulate the gene expression through base-pairing with the $3^{\prime}$-untranslated region ( $3^{\prime}$-UTR) of its target gene and then enhancing the target gene 
degradation or inhibiting the target gene posttranscriptional translation. ${ }^{5}$ It has also been reported that miRNAs play an important role in a variety of physiological processes, including proliferation, differentiation, apoptosis, senescence, etc. ${ }^{4,5}$ Increasing evidence also demonstrate that miRNAs are dysregulated in human disease conditions, such as cancers. ${ }^{6-8}$ The abnormality of miRNA expression in human cancers contributes to the tumor initiation, development, and progression. ${ }^{6-8}$ Recently, several studies also report that miRNAs are abnormally expressed in NPC, ${ }^{9-12}$ and a number of dysregulated miRNAs can regulate NPC cell proliferation, apoptosis, migration, and invasion. ${ }^{12-20}$ These findings suggest that miRNAs play a vital role in NPC tumorigenesis and development. Therefore, further studies are warranted to better elucidate the function of miRNAs in NPC and provide novel therapeutic targets for NPC patients.

In this study, we found that miR-99a is downregulated in NPC cell lines and tissue samples. Overexpression of miR-99a inhibited NPC cell migration and invasion in vitro, and suppressed lung metastasis in vivo. Silencing of miR-99a functioned as an opposite action. $H O X A 1$ was validated as a direct target of miR-99a, and ectopic expression of $H O X A 1$ could rescue the suppressive effect of miR-99a overexpression on NPC cell migration and invasion. Collectively, miR-99a could inhibit NPC metastasis by targeting HOXA1, thus providing a novel potential target for miRNA-based treatment for NPC patients.

\section{Methods}

\section{Cell lines and clinical samples}

Six NPC cell lines, including CNE-1, CNE-2, SUNE-1, C666-1, HNE-1, and HONE-1, were preserved in our laboratory which were originally obtained from Sun Yat-Sen University Cancer Center, and maintained in RPMI-1640 medium (Gibco, Grand Island, NY, USA) supplemented with 10\% fetal bovine serum (Gibco). The immortalized nasopharyngeal epithelial cell line NP69 was purchased from Shanghai Institutes for Biological Sciences, Chinese Academy of Sciences, and cultured in KSFM (Gibco) supplemented with bovine pituitary extract (BD Biosciences, San Diego, CA, USA). All of the cell lines were incubated at $37^{\circ} \mathrm{C}$ in a humidified $5 \% \mathrm{CO}_{2}$ atmosphere. In addition, 4 NPC and its corresponding normal nasopharyngeal epithelial tissue samples were collected from The First Affiliated Hospital of University of South China (Hengyang, People's Republic of China). The Institutional Review Boards of The First Affiliated Hospital of University of South China approved the collection of tissue samples, and written informed consent was obtained from each patient.

\section{RNA isolation and quantitative reverse transcriptase polymerase chain reaction (RT-PCR)}

Total RNA was extracted from NPC cell lines and tissue samples using TRIzol Reagent (Invitrogen, Carlsbad, CA, USA), and reverse-transcribed with M-MLV reverse transcriptase (Promega, Madison, WI, USA). Real-time PCR reactions were done on the PRISM 7900HT sequence detection system (Applied Biosystems, Carlsbad, CA, USA) with Platinum SYBR Green qPCR SuperMix-UDG reagents (Invitrogen). The Bulge-Loop ${ }^{\mathrm{TM}}$ miRNA-specific primers (RiboBio, Guangzhou, People's Republic of China) were used for the detection of miR-99a, while the following primers were used for the detection of HOXA1: forward, TCCTGGAATACCCCATACTTAGC; and reverse, GCA CGACTGGAAAGTTGTAATCC. The U6 or GAPDH was used as controls, and the $2^{-\triangle \Delta \mathrm{CT}}$ equation was used to calculate the relative expression.

\section{Transient transfection}

Cells were seeded into 6-well plates, and transfection was performed using Lipofectamin 2000 reagent (Invitrogen) according to the manufacturer's instructions. The miR-99a mimics and miR-Ctrl were purchased from Genepharma (Suzhou, People's Republic of China), and transfected into CNE-2 and HONE-1 cells. The HOXA1 plasmid or empty vector were purchased from FulenGen (Guangzhou, People's Republic of China), and used to cotransfect CNE-2 and HONE-1 cells, together with miR-99a mimics. After transfection $48 \mathrm{~h}$, the cells were harvested for assays.

\section{Generation of stably transfected cell line}

The sequence of pri-miR-99a was amplified from the human genomic DNA and cloned into the EcoRI and NheI restriction sites of pSin-EF2-puromycin lentiviral plasmid (Addgene, Cambridge, MA, USA). The pSin-EF2-miR-99a or empty vector, together with the lentivirus packaging plasmids psPAX2 and pMD2.G, were used to cotransfect the 293FT cells, and then the lentivirus particles were harvested to infect HONE-1 cells. The stably transfected HONE-1 cells were selected using puromycin and confirmed using quantitative RT-PCR.

\section{Wound healing assay}

CNE-2 and HONE-1 cells were seeded into 6-well plated and cultured until almost totally confluent. After starved for $24 \mathrm{~h}$, artificial wounds were created in the cell monolayer with a sterile $200-\mu \mathrm{L}$ tip, and the floating cells were removed by 
washing with serum-free medium. Respective images were captured at 0 and $48 \mathrm{~h}$ using an inverted microscope.

\section{Transwell migration and invasion assays}

CNE-2 and HONE-1 cells were harvested and resuspended in serum-free medium. $5 \times 10^{4}$ or $1 \times 10^{5}$ cells were added into the Transwell upper chambers, in which the upper surface of the $8 \mu \mathrm{m}$ pore size membrane (Corning, Steuben, NY, USA) coated without or with Matrigel (BD Biosciences). After incubation with 24 or $16 \mathrm{~h}$, the cell which had migrated or invaded through the membrane were fixed, stained, and counted with an inverted microscope.

\section{Lung metastatic colonization model}

Female 4-6 weeks old BALB/c-nu mice were purchased from the Hunan Slac Jingda Laboratory Animal Co., Ltd. (Changsha, People's Republic of China). HONE-1 cells stably expressing miR-99a or vector control were resuspended in phosphate-buffered saline, and $5 \times 10^{5}$ cells were intravenously injected into the tail vein to construct the lung metastatic colonization models. Eight weeks later, the mice were sacrificed, and the lung tissues were paraffin-embedded, cut into serial slides, and one of every 10 slides was stained with hematoxylin and eosin (H\&E). The number of macroscopic or microscopic metastatic nodes was counted. All of the animal research protocols were conducted according to the guidelines of the Experimental Animal Care and Use Committee of the First Affiliated Hospital of University of South China, which also approved this study.

\section{Western blotting}

Total protein was extracted using RIPA buffer containing protease inhibitor cocktail, separated using 9\% sodium dodecyl sulfate-polyacrylamide gel electrophoresis gels, and then transferred to PVDF membranes (Millipore, Billerica, MA, USA). The membranes were incubated with rabbit polyclonal anti-HOXA1 antibody (1:1,000; Abcam, Cambridge, MA, USA) at $4{ }^{\circ} \mathrm{C}$ overnight, followed by goat anti-rabbit secondary antibody (1:5,000; Sigma, St Louis, MO, USA). At last, the bands were visualized with enhanced chemiluminescence, and GAPDH was used as a loading control.

\section{Luciferase reporter assay}

The HOXA1 wild type (WT) 3'-UTR sequence was inserted into the downstream of the firefly luciferase gene in the psiCHECK $^{\mathrm{TM}}$ vector (Promega), and the mutant (MT) plasmid was created by site directed mutagenesis. Then, the HOXA1 WT or MT 3'-UTR reporter plasmids, the p-TK
Renilla plasmid (Promega), together with miR-99a mimics or inhibitor, were used to cotransfect CNE-2 and HONE-1 cells using Lipofectamine 2000 (Invitrogen). After $24 \mathrm{~h}$, the cells were harvested and the Renilla and firefly luciferase activities were determined using the Dual Luciferase Reporter Assay System (Promega).

\section{Statistical analyses}

All experiments were performed in at least 3 independent experiments, and all of the data were presented as the mean \pm standard deviation. Significant differences between groups were analyzed using Student's $t$-test, and $P<0.05$ was defined as statistically significant. All statistical analyses were performed using SPSS 16.0 (SPSS Inc., Chicago, IL, USA).

\section{Results \\ MiR-99a is downregulated in NPC cell lines and clinical specimens}

To know the expression level of miR-99a in NPC, we first tested the miR-99a levels in 6 NPC cell lines and an immortalized nasopharyngeal epithelial cell line NP69 using real-time RT-PCR. The miR-99a expression was significantly downregulated in NPC cell lines than the NP69 cell line (Figure 1A, all $P<0.05$ ). Furthermore, we collected 4 paired NPC and normal nasopharyngeal epithelial tissues, and then tested the expression level of miR-99a using real-time RT-PCR. We found that the expression level of miR-99a was significantly decreased in all NPC tissues compared to its corresponding normal nasopharyngeal epithelial tissues (Figure 1B, all $P<0.05$ ). These findings indicate that miR-99a is downregulated in NPC and it might play a vital role in NPC carcinogenesis and progression.

\section{Overexpression of miR-99a inhibits NPC cell migration and invasion in vitro}

To determine the effect of overexpression of miR-99a on NPC cell migratory and invasive abilities, we transiently transfected CNE-2 and HONE-1 cells with miR-99a mimics or miR controls (miR-Ctrl), and then did wound healing, transwell migration, and invasion assays. As shown in Figure 2A, the wound healing assays displayed that CNE- 2 and HONE-1 cells treated with miR-99a mimics migrated more slowly than cells treated with miR-Ctrl. Furthermore, the transwell migration assays validated that transfection of miR-99a mimics obviously reduced the migratory abilities of CNE-2 and HONE-1 cells (Figure 2B, all $P<0.05$ ). In addition, the transwell invasion assays showed that the invasive abilities of CNE-2 and HONE-1 cells were significantly suppressed 

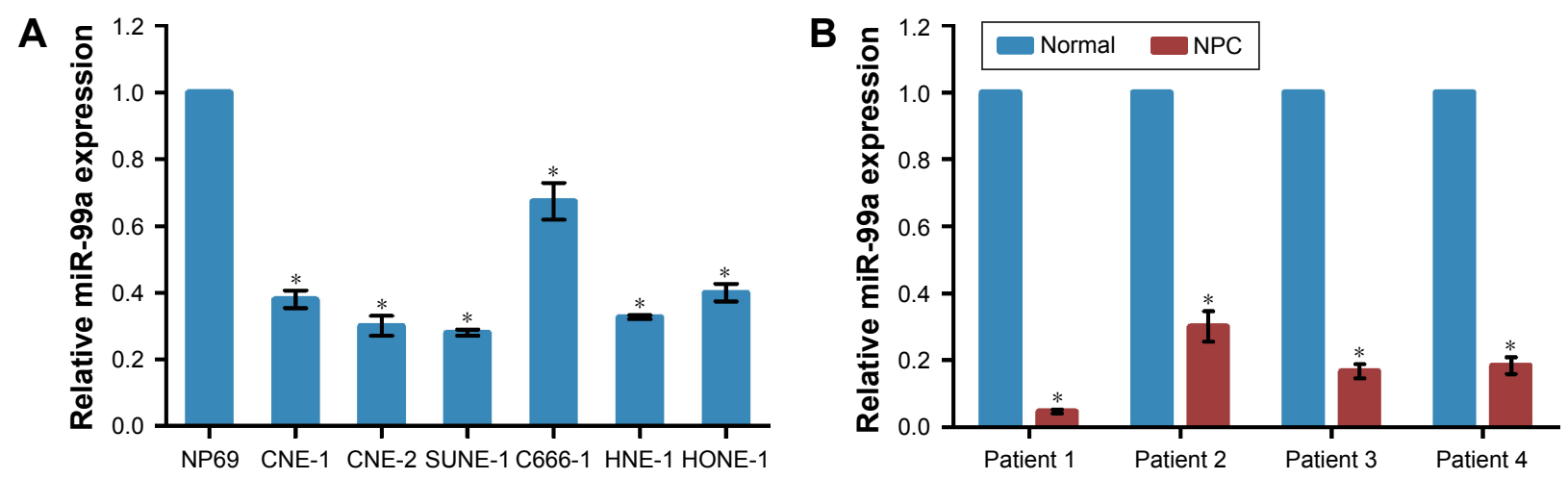

Figure I MiR-99a is downregulated in NPC cell lines and clinical specimens.

Notes: (A) MiR-99a expression in NPC cell lines and the normal nasopharyngeal epithelial cell NP69. (B) MiR-99a expression in 4 paired NPC tissues and normal nasopharyngeal epithelial tissues. MiR-99a expression was normalized to U6 RNA. ${ }^{*} P<0.05$.

Abbreviation: NPC, nasopharyngeal carcinoma.

by miR-99a mimics (Figure 3B, all $P<0.05$ ). These results indicate that the overexpression of miR-99a could inhibit NPC cell migration and invasion in vitro.

\section{Silencing of miR-99a promotes NPC cell migration and invasion in vitro}

To further know the effect of silencing of miR-99a on NPC cell migratory and invasive abilities, we also transiently transfected CNE-2 and HONE-1 cells with miR-99a inhibitor or control (int-Ctrl), and then performed wound healing, transwell migration, and invasion assays. As expected, CNE-2 and HONE-1 cells treated with miR-99a inhibitor migrated more quickly than those treated with int-Ctrl, as determined by the wound healing assays (Figure 3A). Similarly, the transwell migration assays revealed that transfection of miR-99a inhibitor significantly increased the number of migrated cells (Figure 3B, all $P<0.05$ ). The transwell invasion assays also revealed that the invasive abilities of CNE-2 and HONE-1 cells transfected with miR-99a inhibitor was remarkably enhanced (Figure 3C, all $P<0.05$ ). These results demonstrate that the silencing of miR-99a could promote NPC cell migration and invasion in vitro.
A
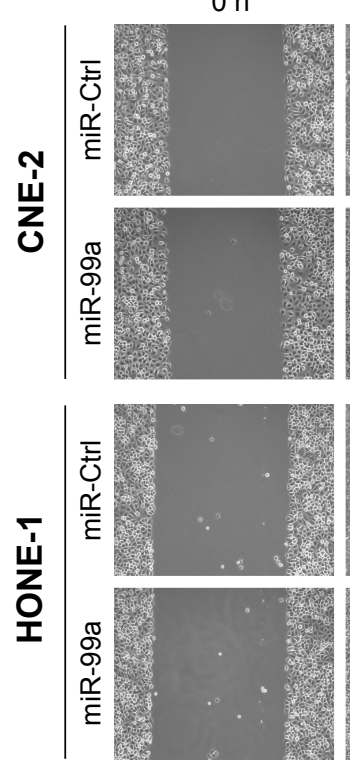

$48 \mathrm{~h}$
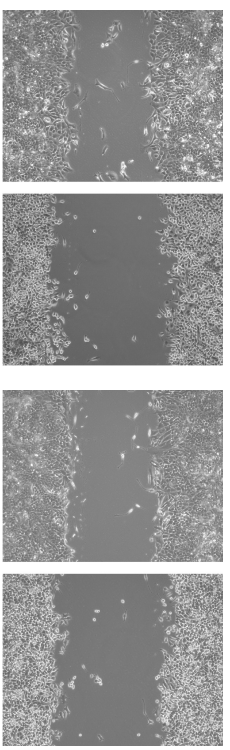

B
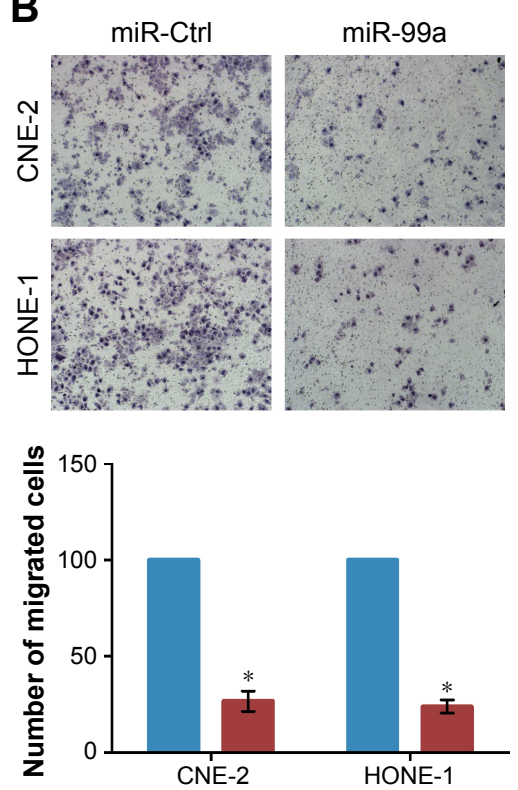

C
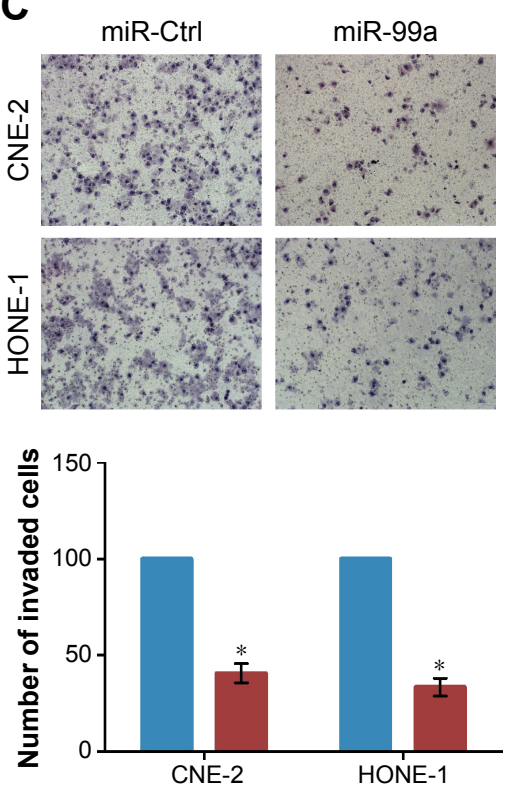

miR-Ctrl miR-99a mimics

Figure 2 Overexpression of miR-99a inhibits nasopharyngeal carcinoma cell migration and invasion in vitro.

Notes: (A-C) Effect of miR-99a overexpression on cell migratory and invasive abilities was measured using wound healing assay (A), transwell migration (B), and invasion (C) assays after transfection of miR-99a mimics or miR control in CNE-2 and HONE-I cells. $* P<0.05$. 
A
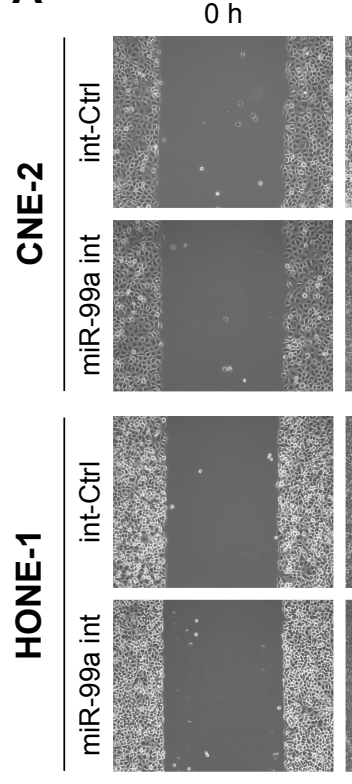

$48 \mathrm{~h}$
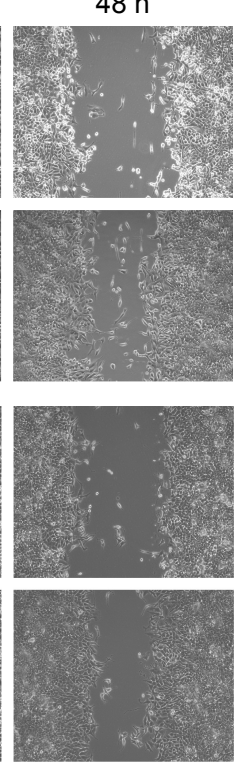

B
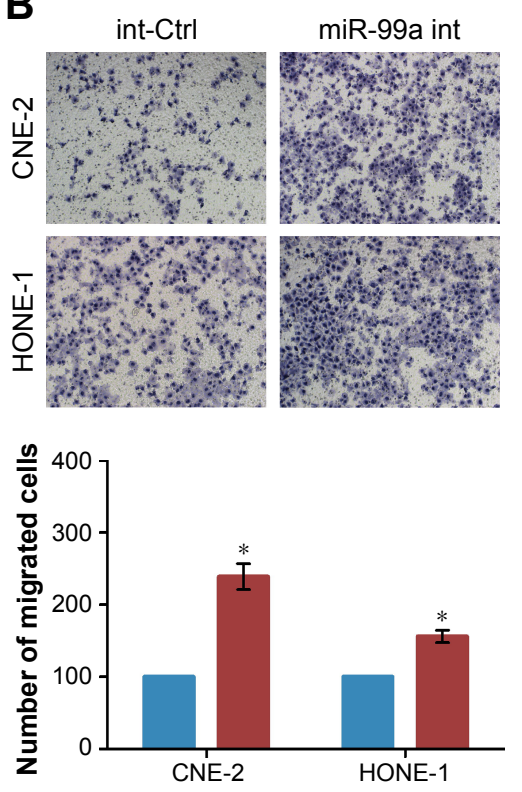

C
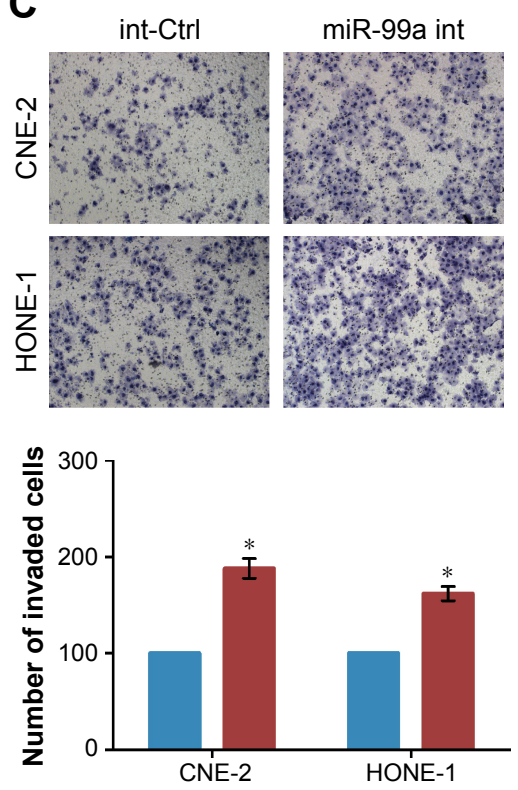

int-Ctrl miR-99a inhibitor

Figure 3 Silencing of miR-99a promotes nasopharyngeal carcinoma cell migration and invasion in vitro.

Notes: (A-C) Effect of miR-99a silencing on cell migratory and invasive abilities was measured using wound healing assay $(\mathbf{A})$, transwell migration $(\mathbf{B})$, and invasion $(\mathbf{C})$ assays after transfection of miR-99a inhibitor or control in CNE-2 and HONE-I cells. *P<0.05.

\section{Overexpression of miR-99a suppresses NPC lung metastasis in vivo}

To investigate whether overexpression of miR-99a affects the metastatic ability of NPC cells, we first established HONE-1 cell lines stably overexpressing miR-99a (lenti-miR-99a) or empty vector (lenti-vector), and then constructed lung metastasis models through injecting cells into the tail veins of nude mice. After 8 weeks, the macroscopic metastatic nodes formed on the surface of lungs in the lenti-miR-99a group were significantly smaller and fewer than those formed in the lenti-vector group (Figure $4 \mathrm{~A}$ and $\mathrm{B}$, all $P<0.05$ ). Furthermore, H\&E staining validated that fewer microscopic metastatic nodes were observed in the lungs of the lenti-miR99a group than in the lenti-vector group (Figure 4C and D, all $P<0.05)$. These results suggest that overexpression of miR-99a could suppress NPC lung metastasis in vivo.

\section{HOXAl is a direct target of miR-99a in NPC}

To explore the mechanism of miR-99a involved in NPC carcinogenesis, we performed bioinformatics analysis using three public databases (TargetScan, miRBase, and MIRDB), and found that the 3 '-UTR of HOXA1 mRNA has one complementary binding site of miR-99a (Figure 5A). Real-time RT-PCR and Western blotting assays revealed that overexpression of miR-99a decreased, while silencing of miR-99a increased the expression of HOXA1 at both the mRNA and protein levels (Figure 5B and $\mathrm{C}$, all $P<0.05$ ), suggesting that HOXA1 may be a target of miR-99a. To validate this, we cloned the WT or MT miR-99a binding site of the HOXA1 3'-UTR into a luciferase reporter vector, and then did luciferase reporter assays in CNE-2 and HONE-1 cells. Overexpression of miR-99a obviously weakened, while silencing of miR-99a enhanced the luciferase activities of the $H O X A 1$ WT reporter plasmids, and this change was not observed in the HOXA1 MT reporter plasmids (Figure 5D, all $P<0.05)$. These results indicate that $H O X A 1$ is a direct target of miR-99a in NPC.

\section{HOXAI is involved in miR-99a-mediated NPC cell migration and invasion}

To further elucidate whether the inhibitory effect of miR-99a on NPC cell migration and invasion was directly mediated by $H O X A 1$, we constructed $H O X A 1$ plasmid (which encode the full length coding sequence of $H O X A 1$ lacking its 3 '-UTR) and control vector, and then used them to transfected CNE-2 and HONE-1 cells together with miR-99a mimics. The wound healing and transwell migration assays showed that overexpression of $H O X A 1$ significantly enhanced the migratory abilities of CNE-2 and HONE-1 cells, which could 
A

Lenti-vector

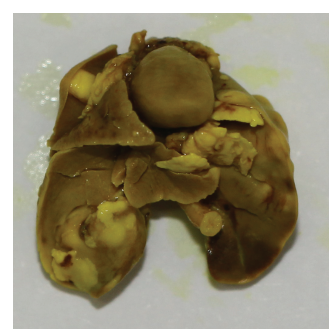

C

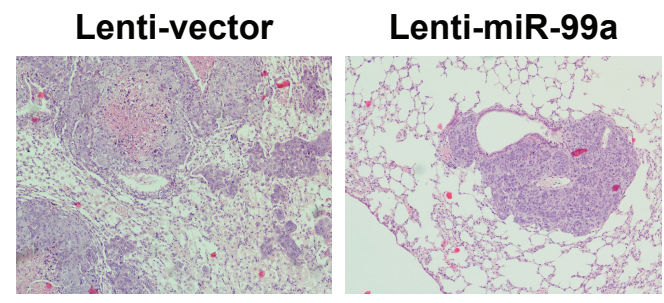

Lenti-miR-99a

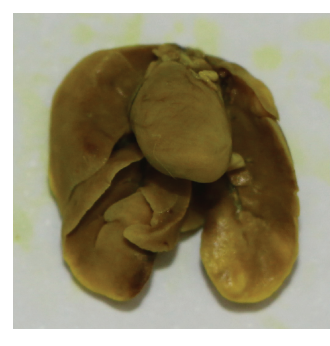

- in 2
B

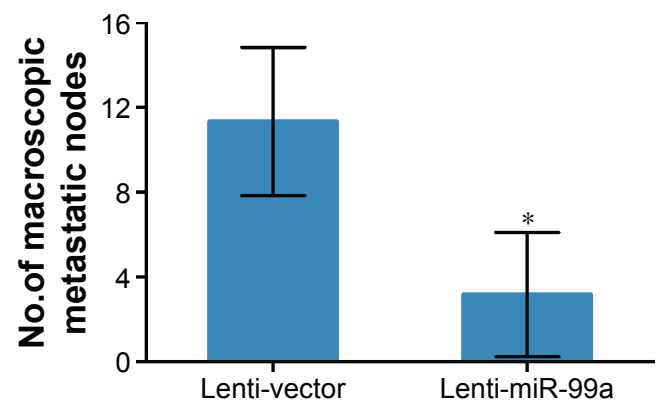

D

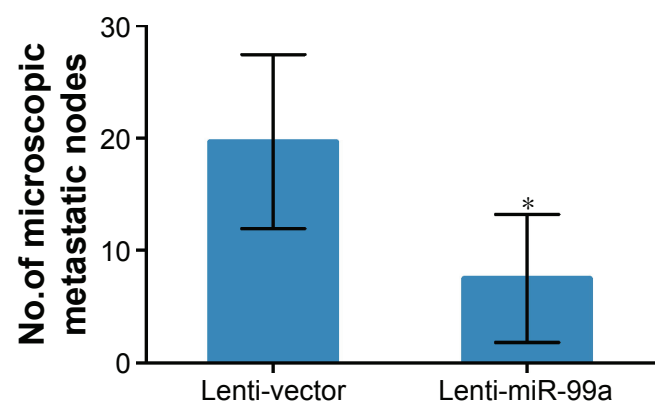

Figure 4 Overexpression of miR-99a suppresses nasopharyngeal carcinoma lung metastasis in vivo.

Notes: (A-D) Lung metastatic colonization models were constructed by injecting HONE-I cells stably overexpressing miR-99a into the tail vein of nude mice. Representative pictures (A) and quantification (B) of macroscopic metastatic tumor nodes formed on the lung surface. Representative pictures (C) and quantification (D) of microscopic metastatic tumor nodes formed in the lung. $* P<0.05$.

be inhibited by miR-99a (Figure 6A and B, all $P<0.05$ ). In addition, the invasive abilities of CNE-2 and HONE-1 cells were increased after overexpression of $H O X A 1$, as determined by the transwell invasion assays (Figure $6 \mathrm{C}$, all $P<0.05)$. These results demonstrate that $H O X A 1$ is a functional target of miR-99a and is involved in miR-99a-mediated NPC cell migration and invasion.

\section{Discussion}

In this study, we found that miR-99a was downregulated in NPC cell lines and clinical specimens. Overexpression of miR-99a inhibited NPC cell migration, invasion, and metastasis in vitro and in vivo. Inversely, silencing of miR-99a promoted NPC cell migration, invasion, and metastasis. HOXA1 was identified and validated as a direct target of miR-99a, and ectopic expression of $H O X A 1$ could rescue the suppressive effect of miR-99a on NPC cell migration and invasion. Collectively, these results demonstrate that miR-99a could suppress NPC metastasis by targeting HOXA1, which would provide novel target for NPC treatment.

Metastasis is responsible for the majority of cancerrelated death worldwide, and distant metastasis is the main cause of death in patient with NPC. ${ }^{3}$ Therefore, great efforts to better understand the molecular mechanisms underlying NPC metastasis are warranted, in order to further improve the prognosis of NPC patients. MiRNAs are small noncoding RNAs that can negatively regulate gene expression, and function as tumor suppressors or oncogenes in the tumorigenesis and progression of human cancers. ${ }^{6-8}$ Several researches have reported that miRNAs are abnormally expressed in NPC tissues, ${ }^{9-12}$ and a number of dysregulated miRNAs are involved in modulating NPC cell growth, proliferation, apoptosis, migration, and invasion. ${ }^{13-20}$ Researches also report that miRNAs can function as prognostic biomarkers and metastatic predictors for NPC patients. ${ }^{21-23}$ All of these findings indicate that miRNAs play a critical role in NPC tumorigenesis and progression. In this study, we found that miR-99a was downregulated in NPC cell lines and tissue samples, indicating that miR-99a might function as a tumor suppressor in NPC development and progression.

Recently, it has been reported that miR-99a was decreased in a variety of human cancers, and might exert its function through affecting tumor cell growth, cell apoptosis, cell cycle, cell proliferation, and cell invasion. ${ }^{24-30} \mathrm{Li}$ et al reported that miR-99a inhibited hepatocellular carcinoma growth and its downregulation was correlated with poor prognosis in patients with hepatocellular carcinoma. ${ }^{24}$ Cui et al demonstrated that miR-99a induced G1-phase cell cycle arrest and suppressed tumorigenicity in renal cell carcinoma. ${ }^{25} \mathrm{Wu}$ et al found that miR-99a could inhibit tumor cell proliferation, migration and 

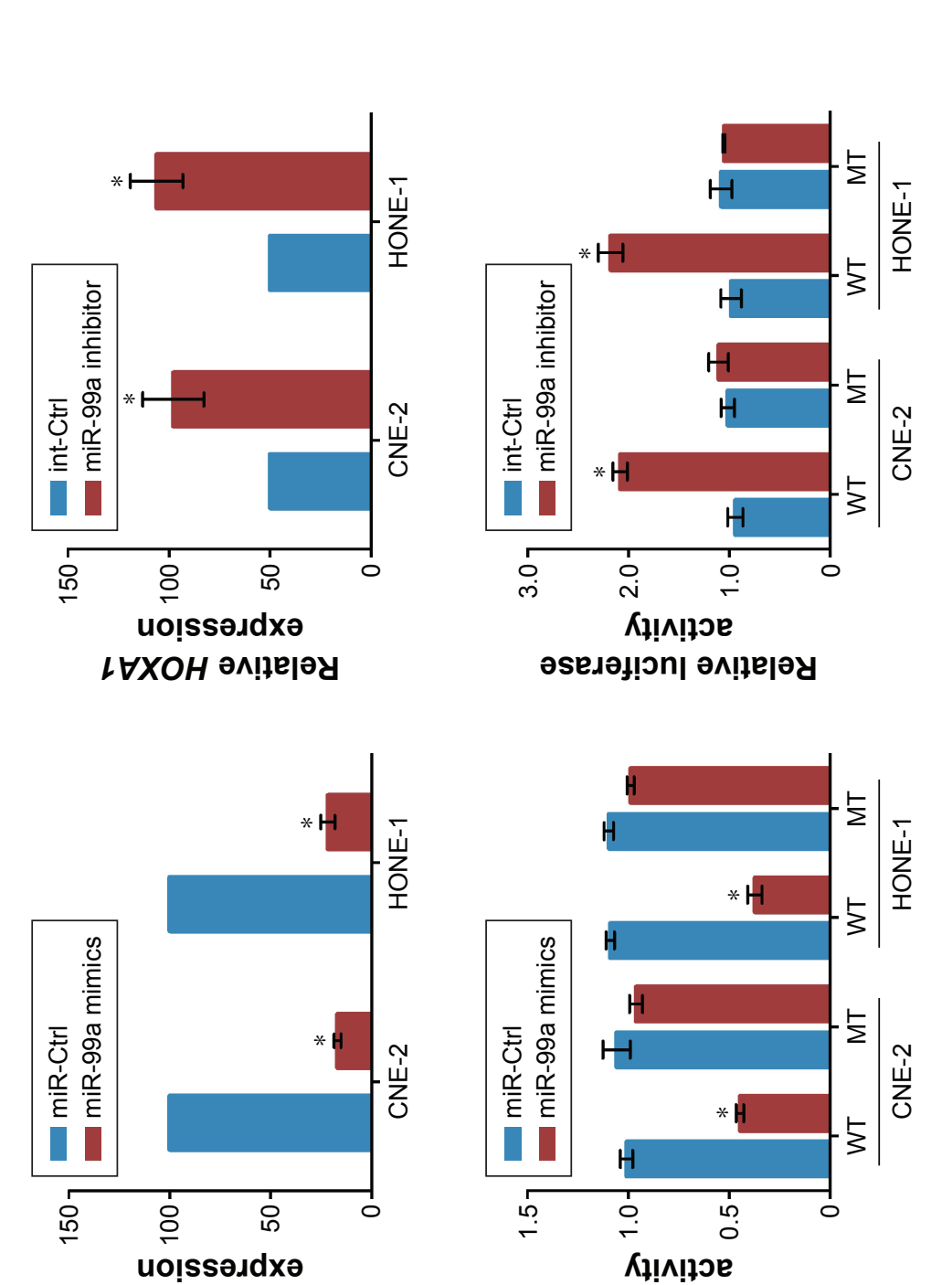

๓

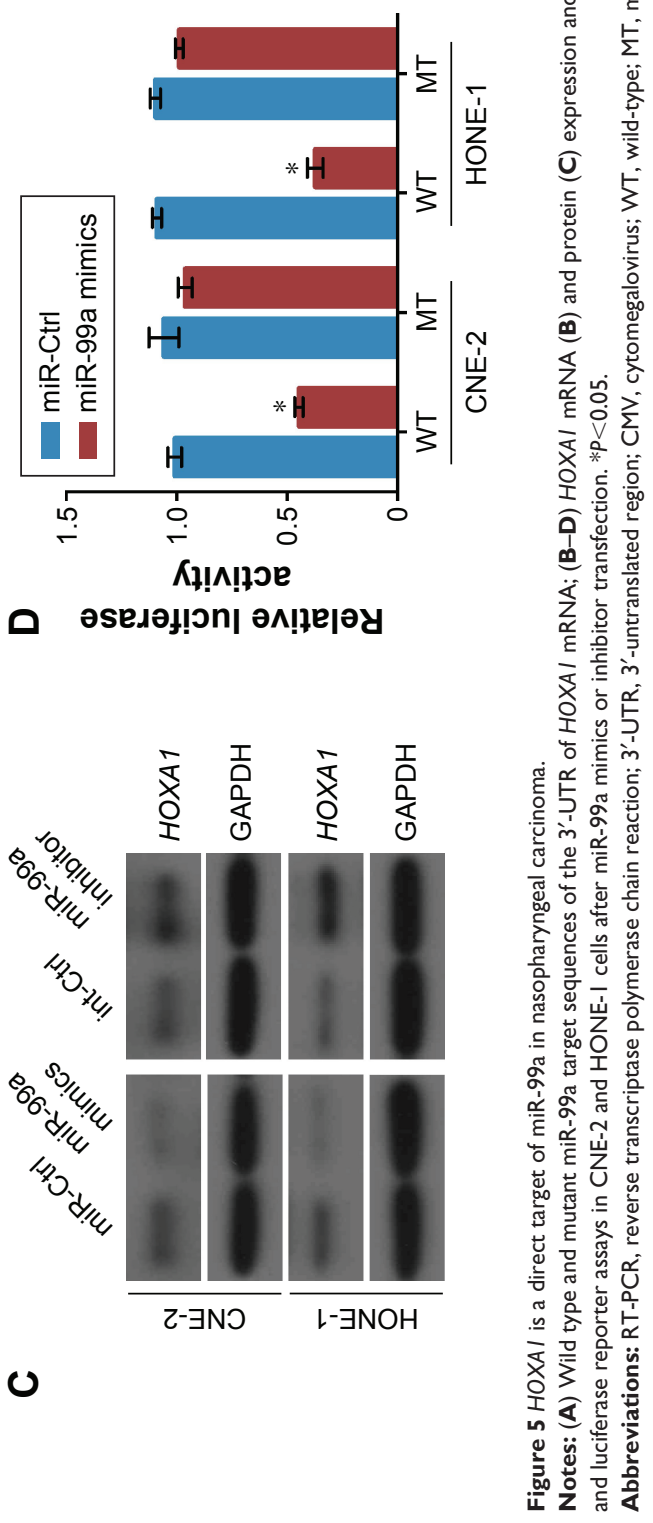


A
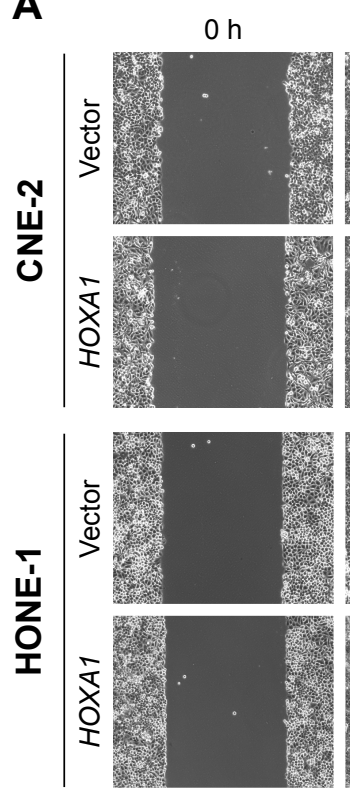

$48 \mathrm{~h}$
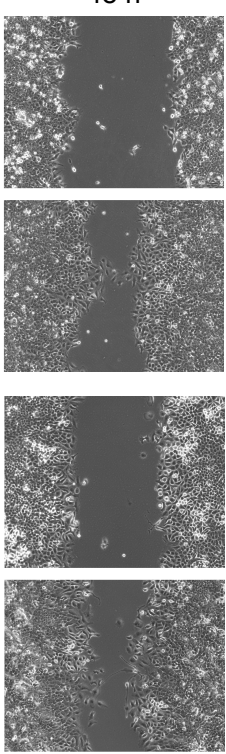

B
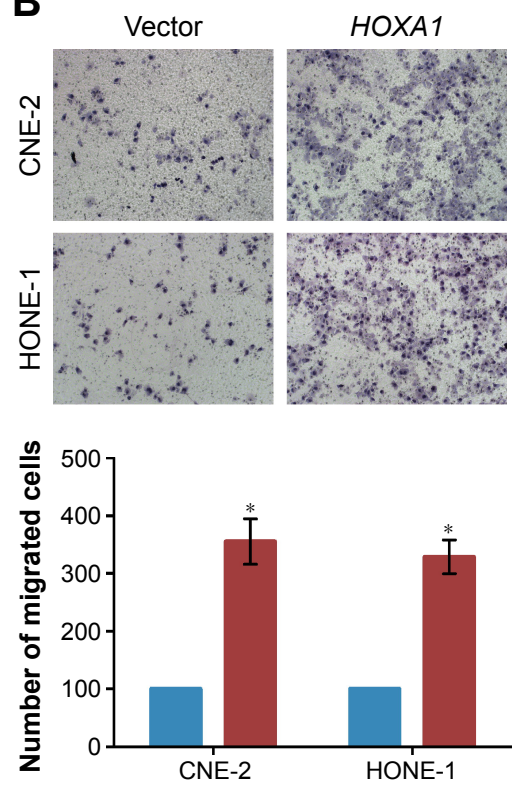

C
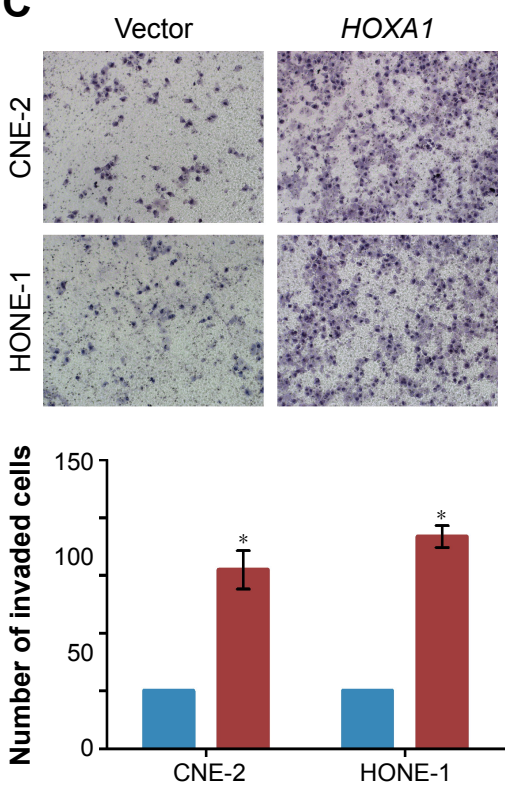

Vector HOXA1

Figure 6 HOXAl is involved in miR-99a-mediated nasopharyngeal carcinoma cell migration and invasion

Notes: (A-C) Effect of HOXAl on cell migratory and invasive abilities was measured using wound healing assay (A), transwell migration (B), and invasion (C) assays after transfection of HOXAI plasmid or vector in CNE-2 and HONE-I cells pre-treated with miR-99a mimics. $* P<0.05$.

invasion in bladder cancer. ${ }^{28}$ Wang et al reported that miR99a inhibited tumor aggressive phenotypes and was associated with poor survival in breast cancer. ${ }^{30}$ In this research, we performed in vitro and in vivo functional studies to determine the function of miR-99a dysregulation in NPC metastasis. We found that overexpression of miR-99a could inhibit cell migration and invasion, while silencing of miR-99a could promote cell migration and invasion in NPC. Our findings indicate that miR-99a functions as a tumor suppressor in NPC metastasis, which enriching the tumor suppressive function of miR-99a in various types of human cancers.

It is well-known that miRNAs function as oncogenes or tumor suppressors through base-paring to the 3'-UTR of their target genes. ${ }^{4,5}$ Great efforts have been made to identify target genes of miR-99a, and several target genes have been verified, including IGF1R, mTOR, SNF2H, TRIB2, IGF3, and FOXA1. ${ }^{24-29}$ Each miRNA can regulate multiple target genes, and each gene can be regulated by multiple miRNAs. ${ }^{4,5}$ Therefore, in this study, we tried to search for novel targets of miR-99a using three public available databases, and found that HOXA1 might be a potential target of miR-99a. Real-time RTPCR and Western blotting found that overexpression of miR99a decreased, whereas silencing of miR-99a increased the expression of HOXA1 at both the mRNA and protein levels. Furthermore, luciferase reporter assay verified that HOXA1 was a direct target of miR-99a in NPC. Similarly, HOXA1 was also confirmed as a direct target of miR-99a in breast cancer. ${ }^{30}$ As expected, functional studies demonstrated that $H O X A 1$ was involved in miR-99a-mediated NPC cell migration and invasion, which indicating that $H O X A 1$ was a functional target of miR-99a in NPC, and miR-99a/HOXA1 might be a common treatment targets in NPC and breast cancers.

\section{Conclusion}

Our findings found that miR-99a functioned as a tumor suppressor in NPC. Overexpression of miR-99a could inhibit NPC cell migration and invasion, whereas silencing of miR99a could promote NPC cell migration and invasion. HOXA1 was predicted and verified as a direct target of miR-99a, and was involved in miR-99a-medaited NPC cell migration and invasion. Therefore, the miR-99a/HOXA1 signaling pathway would provide novel insights into NPC metastasis and provide novel therapeutic targets for NPC patients.

\section{Acknowledgments}

This work was supported by grant from the Science and Technology Project of Hengyang City, People's Republic of China (No. 2013KJ50). The funders had no role in the study design, data collection, analysis, decision to publish or the preparation of the manuscript. 


\section{Author contributions}

All authors contributed toward data analysis, drafting and critically revising the paper and agree to be accountable for all aspects of the work.

\section{Disclosure}

The authors report no conflicts of interest in this work.

\section{References}

1. Torre LA, Bray F, Siegel RL, Ferlay J, Lortet-Tieulent J, Jemal A. Global cancer statistics, 2012. CA Cancer J Clin. 2015;65(2):87-108.

2. Lee AW, Ma BB, Ng WT, Chan AT. Management of nasopharyngeal carcinoma: current practice and future perspective. J Clin Oncol. 2015; 33(29):3356-3364.

3. Razak AR, Siu LL, Liu FF, Ito E, O'Sullivan B, Chan K. Nasopharyngeal carcinoma: the next challenges. Eur J Cancer. 2010;46(11): 1967-1978.

4. Bartel DP. MicroRNAs: genomics, biogenesis, mechanism, and function. Cell. 2004;116(2):281-297.

5. Bartel DP. MicroRNAs: target recognition and regulatory functions. Cell. 2009;136(2):215-233.

6. Lu J, Getz G, Miska EA, et al. MicroRNA expression profiles classify human cancers. Nature. 2005;435(7043):834-838.

7. Esquela-Kerscher A, Slack FJ. Oncomirs-microRNAs with a role in cancer. Nat Rev Cancer. 2006;6(4):259-269.

8. Croce CM. Causes and consequences of microRNA dysregulation in cancer. Nat Rev Genet. 2009;10(10):704-714.

9. He ML, Luo MX, Lin MC, Kung HF. MicroRNAs: potential diagnostic markers and therapeutic targets for EBV-associated nasopharyngeal carcinoma. Biochim Biophys Acta. 2012;1825(1):1-10.

10. Bruce JP, Liu FF. MicroRNAs in nasopharyngeal carcinoma. Chin J Cancer. 2014;33(11):539-544.

11. Chen HC, Chen GH, Chen YH, et al. MicroRNA deregulation and pathway alterations in nasopharyngeal carcinoma. Br J Cancer. 2009; 100(6):1002-1011.

12. Li T, Chen JX, Fu XP, et al. microRNA expression profiling of nasopharyngeal carcinoma. Oncol Rep. 2011;25(5):1353-1363.

13. Lu J, He ML, Wang L, et al. MiR-26a inhibits cell growth and tumorigenesis of nasopharyngeal carcinoma through repression of EZH2. Cancer Res. 2011;71(1):225-233.

14. Yi C, Wang Q, Wang L, et al. MiR-663, a microRNA targeting p21 (WAF1/CIP1), promotes the proliferation and tumorigenesis of nasopharyngeal carcinoma. Oncogene. 2012;31(41):4421-4433.

15. Liu N, Tang LL, Sun Y, et al. MiR-29c suppresses invasion and metastasis by targeting TIAM1 in nasopharyngeal carcinoma. Cancer Lett. 2013; 329(2):181-188

OncoTargets and Therapy

\section{Publish your work in this journal}

OncoTargets and Therapy is an international, peer-reviewed, open access journal focusing on the pathological basis of all cancers, potential targets for therapy and treatment protocols employed to improve the management of cancer patients. The journal also focuses on the impact of management programs and new therapeutic agents and protocols on

Submit your manuscript here: http://www.dovepress.com/oncotargets-and-therapy-journal
16. Peng XH, Huang HR, Lu J, et al. MiR-124 suppresses tumor growth and metastasis by targeting Foxq1 in nasopharyngeal carcinoma. Mol Cancer. 2014;13:186.

17. Li YQ, Ren XY, He QM, et al. MiR-34c suppresses tumor growth and metastasis in nasopharyngeal carcinoma by targeting MET. Cell Death Dis. 2015;6:e1618.

18. Xu YF, Mao YP, Li YQ, et al. MicroRNA-93 promotes cell growth and invasion in nasopharyngeal carcinoma by targeting disabled homolog-2. Cancer Lett. 2015;363(2):146-155.

19. Zhao M, Luo R, Liu Y, et al. miR-3188 regulates nasopharyngeal carcinoma proliferation and chemosensitivity through a FOXO1-modulated positive feedback loop with mTOR-p-PI3K/AKT-c-JUN. Nat Commun. 2016;7:11309.

20. Li Y, Tang X, He Q, et al. Overexpression of mitochondria mediator gene TRIAP1 by miR-320b loss is associated with progression in nasopharyngeal carcinoma. PLoS Genet. 2016;12(7):e1006183.

21. Liu N, Chen NY, Cui RX, et al. Prognostic value of a microRNA signature in nasopharyngeal carcinoma: a microRNA expression analysis. Lancet Oncol. 2012;13(6):633-641.

22. Liu N, Cui RX, Sun Y, et al. A four-miRNA signature identified from genome-wide serum miRNA profiling predicts survival in patients with nasopharyngeal carcinoma. Int J Cancer. 2014;134(6):1359-1368.

23. Bruce JP, Hui AB, Shi W, et al. Identification of a microRNA signature associated with risk of distant metastasis in nasopharyngeal carcinoma. Oncotarget. 2015;6(6):4537-4550.

24. Li D, Liu X, Lin L, et al. MicroRNA-99a inhibits hepatocellular carcinoma growth and correlates with prognosis of patients with hepatocellular carcinoma. $J$ Biol Chem. 2011;286(42):36677-36685.

25. Cui L, Zhou H, Zhao H, et al. MicroRNA-99a induces G1-phase cell cycle arrest and suppresses tumorigenicity in renal cell carcinoma. $B M C$ Cancer. 2012;12:546.

26. Mueller AC, Sun D, Dutta A. The miR-99 family regulates the DNA damage response through its target SNF2H. Oncogene. 2013; 32(9):1164-1172.

27. Xin JX, Yue Z, Zhang S, et al. miR-99 inhibits cervical carcinoma cell proliferation by targeting TRIB2. Oncol Lett. 2013;6(4):1025-1030.

28. Wu D, Zhou Y, Pan H, Zhou J, Fan Y, Qu P. microRNA-99a inhibiting cell proliferation, migration and invasion by targeting fibroblast growth factor receptor 3 in bladder cancer. Oncol Lett. 2014; 7(4):1219-1224.

29. Drayton RM, Peter S, Myers K, et al. MicroRNA-99a and 100 mediated upregulation of FOXA1 in bladder cancer. Oncotarget. 2014; 5(15):6375-6386

30. Wang X, Li Y, Qi W, et al. MicroRNA-99a inhibits tumor aggressive phenotypes through regulating HOXA1 in breast cancer cells. Oncotarget. 2015;6(32):32737-32747. 